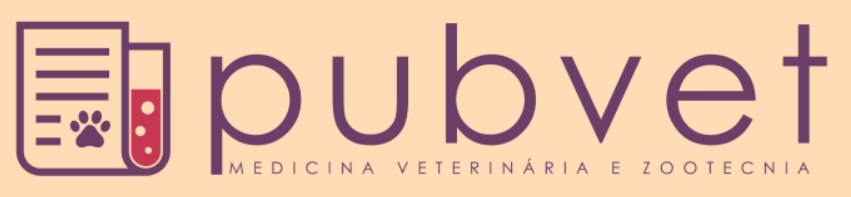

https://doi.org/10.31533/pubvet.v12n11a213.1-8

\title{
Alimentação de cães e gatos cardiopatas
}

\author{
Jéssica de Carvalho Pantoja ${ }^{*} \bullet$, Ícaro dos Santos Cabral ${ }^{2} \bullet$, Thaiza Santos Farias ${ }^{30}$, \\ Thaís Emanuely dos Santos Amaral ${ }^{30}$, Cristiane Rebouças Barbosa ${ }^{3 \bullet}$ \\ ${ }^{1}$ Bacharela em Ciências Agrárias - Produção Animal. Universidade Federal do Oeste do Pará. Santarém, Pará, Brasil. \\ ${ }^{2}$ Professor adjunto da Universidade Federal do Pará, Instituto de Biodiversidade e Florestas. Santarém, Pará, Brasil. \\ ${ }^{3}$ Discentes do curso de Zootecnia da Universidade Federal do Oeste do Pará. Santarém, Pará, Brasil. \\ *Autor para correspondência, jessicka.carvalho17@gmail.com
}

RESUMO. Atualmente se reconhece a importância do manejo alimentar no auxílio de prevenção de algumas doenças e muita das vezes da sua cura. Com isso, objetivou-se avaliar na literatura o manejo na alimentação de cães e gatos condicionados ao problema cardíaco. Problemas do coração são uma das maiores causas de morte em alguns pets, facilitando a falência de outros órgãos e ainda podendo provocar outros tipos de doença. De acordo com as pesquisas foi observado um avanço na dieta desses animais, sendo que o comércio hoje disponibiliza alimentação específica para cardiopatas, com níveis adequados de nutrientes essenciais. É importante observar as quantidades principalmente de sódio, magnésio e taurina, além de outros considerados importantes para a manutenção de cães e gatos. Diversos trabalhos disponibilizam ainda outras opções como a dieta caseira, no entanto, tendo auxílio de um especialista em nutrição.

Palavras chave: dieta, doenças, nutrição

\section{Feeding of dogs and cats with heart disease}

ABSTRACT. Now recognizes the importance of food aid management of prevention of some diseases and much of the time of your healing. With that, the objective of evaluating the management literature on dogs and cats conditioned power to the heart condition. Heart problems are one of the leading causes of death in some pets, making the failure of other organs and causing still other types of disease. According to the researchers noted an improvement in the diet of these animals, being that trade today offers specific feed for cardiac patients, with adequate levels of essential nutrients. It is important to note the quantities mainly sodium, magnesium and taurine, and others considered important for maintenance of cats and dogs. Several papers offer still other options like the homemade diet, however, having a nutrition specialist.

Keywords: diet, diseases, nutrition

\section{Alimentación de perros y gatos cardiópatas}

RESUMEN. Actualmente se reconoce la importancia del manejo alimentario en la ayuda de prevención de algunas enfermedades y muchas veces de su cura. Con ello, se objetivó evaluar en la literatura el manejo en la alimentación de perros y gatos condicionados al problema cardíaco. Los problemas del corazón son una de las mayores causas de muerte en algunas mascotas, facilitando la falla de otros órganos y aun pudiendo provocar otros tipos de enfermedad. De acuerdo con las investigaciones se observó un avance en la dieta de estos animales, siendo que el comercio hoy dispone de alimentación específica para cardiópatas, con niveles adecuados de nutrientes esenciales. Es importante observar las 
cantidades principalmente de sodio, magnesio y taurina, además de otros considerados importantes para el mantenimiento de perros y gatos. Diversos trabajos también ofrecen otras opciones como la dieta casera, sin embargo, ayudando a un especialista en nutrición.

Palabras clave: dieta, enfermedades, nutrición

\section{Introdução}

A alimentação de cães e gatos passou por uma evolução nas últimas décadas. Na década de 80 , a maioria dos pets ainda eram alimentados com restos de comida de seus proprietários, e poucas indústrias de rações existiam e investiam no Brasil. Neste ponto, dois fatores contribuíram para a expansão do segmento; o poder aquisitivo das populações dos grandes centros aumentou e os padrões de consumo se sofisticaram. Por outro lado, a evolução dos hábitos em favor dos alimentos industriais está associada a um conjunto de fatores cada vez mais difundidos: alimentação sadia, equilibrada e com grande variedade de produtos disponíveis no mercado e, principalmente, a praticidade.

Antigamente o objetivo da nutrição de cães e gatos era apenas proporcionar calorias, proteínas, gorduras, vitaminas e minerais. Atualmente, avanços ocorreram nessa área e pesquisadores descobriram que a nutrição também pode desempenhar um papel importante na gestão de doenças, incluindo a doença cardíaca (Freeman, 2000a). Segundo Carciofi \& Jeremias (2010) pesquisas científicas relacionadas à nutrição de animais de companhia, principalmente nos últimos 10 anos, deixaram de focar a dicotomia necessidades mínimas e teores máximos, sobretudo quanto ao estabelecimento das recomendações nutricionais. Além disso, a adequação da composição nutricional, matriz de ingredientes, processamento do alimento, entre outros, às necessidades específicas de cada estágio de vida, à condição fisiológica, ao estilo de vida e às diversas condições fisiopatológicas que acometem estes animais têm ocupado os temas principais de pesquisa (Carciofi \& Jeremias, $\underline{2010}$ ). Hoje se reconhece a importância do manejo alimentar para algumas doenças que induzem alterações metabólicas e funcionais específicas (Zaine et al., 2014). Para Hand et al. (2010), a dieta é formulada de modo a apresentar composição nutricional que melhor se adeque às modificações metabólicas induzidas pela doença e é ferramenta importante, que vem sendo largamente empregada.

Assim, conhecer o manejo alimentar empregado aos cães e gatos cardiopatas é fundamental para o desenvolvimento de uma dieta específica. Diante disso, objetivou-se avaliar na literatura o manejo na alimentação desses animais condicionados ao problema cardíaco.

\section{Importância do sistema cardiovascular}

O coração é o órgão responsável por bombear o sangue pelo corpo. Sem esse bombeamento não há transporte de oxigênio e alimento para as células, o que pode causar a morte. O sangue dos pets, assim como o dos humanos é transportado exclusivamente dentro de vasos sanguíneos: as artérias, veias, capilares etc.

O coração é formado por músculo cardíaco, e é composto por quatro câmaras (2 átrios e 2 ventrículos). A função do sistema cardiovascular é manter a pressão arterial e o fluxo sanguíneo normal, enquanto mantém normais as pressões do sangue venoso e dos capilares. A manutenção da pressão do sangue arterial e do débito cardíaco é necessária para providenciar uma adequada oxigenação do fluxo sanguíneo e distribuição dos nutrientes vitais para os tecidos (Tilley \& Goodwin, 2002).

De acordo com Evangelista (2005), a importância dos alimentos não é medida apenas pelo desempenho e valor dos seus constituintes, mas também pelos possíveis danos que a ausência ou excesso destes podem acarretar ao organismo animal. As substâncias para que cheguem ao seu destino e atinjam o metabolismo precisam ser conduzidas. E quem assume este papel importante é o sistema cardiovascular, uma vez que o sangue é um meio de condução e atua como veículo para a maioria dos processos homeostáticos, desempenhando papel em quase todas as funções fisiológicas e tornando compreensível a importância do coração. Randall et al. (2000) explicam que o órgão funciona como uma bomba de pressão que ejeta o sangue para todas as estruturas corporais, permitindo, assim, a chegada dos nutrientes em cada célula do organismo. Eque a partir daí entende-se, a necessidade da manutenção da integridade do sistema cardiovascular cujas funções desempenhadas são, de fato, essenciais. As células do músculo cardíaco em condições normais operam quase que exclusivamente sob um sistema metabólico 
aeróbico que fornece suprimento constante de ligações de fosfato de alta energia para realização do trabalho químico e mecânico. Azevedo et al. (2005) citam que, geralmente o principal combustível para a contratilidade miocárdica são os ácidos graxos livres, sendo ainda contribuintes a glicose e o lactato, ao passo que os aminoácidos, as cetonas e o piruvato contribuem em escala bem menor.

\section{Doenças cardíacas}

As doenças cardíacas são cada vez mais comuns em cães e gatos. Isto ocorre porque a expectativa de vida desses animais tem aumentado nos últimos anos, principalmente devido à melhor qualidade dos serviços veterinários atualmente prestados. As cardiopatias podem ser congênitas ou adquiridas, e quando corretamente diagnosticadas e tratadas, podem permitir ao animal uma excelente qualidade de vida. As alterações adquiridas são mais frequentes em cães idosos e são geralmente causadas por alterações nas válvulas cardíacas ou por alterações no músculo cardíaco (miocárdio) (Nelson \& Couto, $\underline{2015}$ ).

As cardiopatias podem ocorrer tanto no próprio músculo do coração (cardiomiopatias), nas válvulas cardíacas (valvulopatias) ou nas artérias que irrigam o coração (coronariopatias). As cardiomiopatias fazem parte do grupo heterogêneo de doenças do músculo cardíaco vinculadas a diversas etiologias e várias expressões fenotípicas (Silveira et al., 2015). Segundo Saad \& França (2010), animais cardiopatas têm como característica intolerância ao exercício, cansaço inexplicável, diminuição do apetite, entre outros sintomas.

Dentre as principais doenças a Insuficiência Cardíaca (IC) está entre as mais graves e que ocorre com maior frequência nos pets (Nelson \& Couto, 2015). Trata-se de uma síndrome mecânica e neuroendócrina em que o coração falha como bomba, o que resulta em estase de sangue nos pulmões e no sistema venoso, em fadiga e intolerância ao esforço e em redução de longevidade. Do ponto de vista puramente mecânico, a IC é definida como uma síndrome clínica resultante de qualquer desordem cardíaca estrutural ou funcional que causa prejuízo do enchimento ventricular ou da ejeção de sangue (Chawla et al., 2014). A insuficiência cardíaca ocorre quando o sangue que retorna ao coração não pode ser bombeado com velocidade compatível com as demandas metabólicas do corpo. A IC tem muitas etiologias, desde aquelas que impõem sobrecargas mecânicas sistólicas de volume ou de pressão sobre o coração, as que reduzem a contratilidade do miocárdio, as quem interferem na repleção ventricular, ou aumentam as exigências metabólicas sistêmicas, até as que comprometem a função cardíaca (Nelson \& Couto, 2015). A síndrome clínica de insuficiência cardíaca é uma manifestação de consequências circulatórias. Essas consequências resultam vários sistemas neuro-humorais, que são importantes para a manutenção da função cardiovascular, mas quando ativados de forma crônica têm efeitos deletérios no sistema cardiovascular (Santos Júnior et al., 2007). As alterações neuro-humorais na IC incluem o aumento do tônus nervoso simpático, a ativação do sistema reninaangiotensina-aldosterona (SRAA) e a liberação da vasopressina, hormônio antidiurético (ADH) (Little et al., 2005).

De acordo com Darke et al. (2000), o coração insuficiente pode ser estimulado a contrair mais vigorosamente pelo aumento do tônus simpático e pela dilatação cardíaca (efeito Frank-Starling), mas essa resposta é ótima apenas se o miocárdio estiver saudável. Morais (2005) cita que o aumento da concentração de noradrenalina diminui a regulação dos receptores $\beta$ adrenérgicos.

O débito cardíaco diminuído, durante a IC, ativa o sistema renina-angiotensina, pela estimulação direta do $\beta$-adrenorreceptor e pela diminuição do fluxo renal no aparelho justaglomerular dos rins. A angiotensina II, ativada pela enzima conversora da angiotensina (ECA) é um potente vasoconstritor, estimula a liberação de aldosterona, do hormônio antidiurético (ADH), aumenta a atividade simpática, promove constrição na artéria renal eferente e estimula a sede (Teerlink, 1996).

A IC representa um grupo heterogêneo de síndromes que levam a alterações estruturais (hipertrofia, fibrose) e funcionais (rigidez miocárdica e relaxamento incompleto de unidades contráteis). A sequência anormal de expressão de genes, as sinalizações celulares e o estresse oxidativo e outros fatores são um elo comum, o qual é responsável por um exacerbado trabalho de reparação (fibrose). Estas transformações estruturais e funcionais tornam o coração mais vulnerável a arritmias e falha de bomba cardíaca (Husain-Syed et al., 2015). Em pequenos animais 
a IC é normalmente um problema crônico (Morais, 2005). Borges et al. (2016), em um estudo retrospectivo de 10.805 cães com alterações cardiovasculares atendidos em uma clínica, observaram que $100 \%$ dos animais apresentavam histórico e sinais clínicos compatíveis com insuficiência cardíaca. Sendo que, $67,7 \%$ enquadravam-se na fase de senilidade, com idade máxima de 15 anos.

Para tratar a insuficiência cardíaca utilizam-se medicamentos ou por meio de dieta controlada. A alimentação controlada é feita por meio da utilização de ração industrializada, que tem principalmente o sódio controlado para que não ocorra hipertensão. Se a doença for avançada, precisa-se cortar totalmente o sal. O paciente que apresentar IC necessita manter uma dieta controlada pelo resto da vida (Saad \& França, 2010). O alimento industrializado deve conter taurina e L-carnitina, pois esses são indispensáveis para o bom funcionamento do miócitos e favorecem a contratilidade cardíaca (Saad \& França, 2010) e também deve conter quantidades aumentadas de potássio e vitaminas B para compensar as perdas urinárias excessivas destes componentes.

De acordo com Nelson \& Couto (2015) a gravidade da insuficiência cardíaca pode ser classificada conforme o sistema proposto pelo International Small Animal Cardiac Health Council (ISACHC), em Ia (paciente assintomático sem cardiomegalia ou arritmias); Ib (Paciente assintomático e compensado, com cardiomegalia ou arritmias); II (paciente sintomático com insuficiência cardíaca leve a moderada); IIIa (Insuficiência cardíaca avançada) e IIIb (IC avançada, internação recomendada).

Entre as enfermidades que acometem o coração destaca-se também a cardiomiopatia dilatada que é caracterizada pela insuficiência miocárdica sistólica. Sua causa na maior parte dos casos individuais em cães e gatos, bem como em outras espécies, continua desconhecida. Contudo, tal doença tem sido associada a uma variedade de agressões potencias ao miocárdio, incluindo aquelas provocadas por mutações genéticas, agentes infecciosos, defeitos bioquímicos das mitocôndrias e proteínas, toxinas, mecanismos imunológicos e deficiências nutricionais (Morais, 2005). Castro et al. (2009) avaliaram as principais cardiopatias diagnosticadas em cães. Dos 854 cães estudados, $750(88 \%)$ apresentaram alguma alteração cardíaca no exame ecodopplercardiográfico, sendo a doença valvular adquirida a de maior ocorrência (77\%), enquanto $104(12 \%)$ foram considerados normais quanto à avaliação cardíaca.

Um dos aspectos mais importantes do cuidado de cães com falha cardíaca é manter o peso e a condição corporal num patamar aceitável (Slupe et al., 2008). Sendo assim, a caquexia cardíaca ou perda de massa magra, é uma síndrome comum em cães com IC. Dentre as cardiopatias congênitas, temos a persistência do ducto arterioso, também denominada de ducto arterioso patente que é o defeito cardíaco congênito mais comum em cães (Assumpção et al., 2012). Devido à cronicidade e progressão características da IC a reavaliação periódica é essencial. O tratamento deve ser ajustado com o evoluir da doença, alterando doses, adicionando ou retirando fármacos, modificando o estilo de vida ou a dieta (Nelson \& Couto, 2015).

\section{Manejo nutricional nos cardiopatas}

Nelson \& Couto (2015) citam que as cardiopatias são frequentemente encontradas em cães e gatos idosos. Depois de diagnosticada devese ficar atento para identificar problemas de saúde concomitantes. Carr (2008) afirma que um exemplo é o cão que possui insuficiência cardíaca e renal.

Segundo Freeman (2009) durante muito tempo o papel da nutrição no manejo da doença cardíaca consistiu primariamente da alimentação com dieta com baixos teores de sódio. Atualmente, sabe-se que a severa restrição de sódio não é necessária em todos os animais com doença cardíaca. $\mathrm{O}$ autor ainda afirma que pesquisas atuais demonstram que a nutrição pode modular a cardiopatia através da diminuição de sua progressão e da minimização do número de medicamentos necessários, promovendo qualidade de vida ou, em casos raros, atuando na cura da doença. Portanto, atenção à dieta em todos os estágios da doença cardíaca é essencial para a otimização dos cuidados aos pacientes cardiopatas. Além disso, por vários anos foi recomendado que animais com insuficiência cardíaca fossem alimentados com dietas com baixos níveis de proteína para proteger a função renal, uma vez que as doenças cardíacas muitas vezes culminam com prejuízo na função renal. No entanto, a restrição proteica pode ser prejudicial no que diz respeito à perda de massa magra e desnutrição, aumentando assim o risco de caquexia cardíaca e intolerância ao exercício (Chetboul \& Biourge, 2008). Roudebush \& Keene 
(2010) afirmam que, como no curso da doença cardíaca os pacientes podem perder a capacidade de excretar o excesso de sódio, preconiza-se desde o primeiro sinal da doença a introdução de alimentos com reduzidos níveis desse elemento, visando à prevenção da função renal.

Os mecanismos neuro-humorais ativados na IC levam à retenção de sódio, cloro e água nos nefróns de forma a aumentar o volume sanguíneo circulante. Classicamente recomenda-se uma dieta baixa em sal a doentes cardíacos de forma a reduzir o cloro e sódio totais sanguíneos e contrariar esses mecanismos. No entanto, a administração crônica de dietas baixas em sal leva a uma maior conservação de sódio e cloro pela ativação do SRAA (sistema renina-angiotensinaaldosterona), levando a uma maior progressão da IC.

A AAFCO (Associação americana de controle de alimentos) preconiza o mínimo de sódio de 20 $\mathrm{mg} / 100 \mathrm{kcal}$ para cães e $50 \mathrm{mg} / 100 \mathrm{kcal}$ para gatos, não é recomendada para os estágios iniciais da cardiopatia Recomenda-se leve restrição em animais assintomáticos, ou seja, em estágio Ia descrito pelo Internacional Small Animal Cardiac Health Council (ISACHC), com quantidades inferiores a $100 \mathrm{mg}$ de sódio/100 kcal e nos cães pertencentes à classe I-b, ou seja, assintomáticos, porém com alterações em exames complementares que indiquem remodelamento cardíaco, recomenda-se menos de $80 \mathrm{mg}$ de sódio/100 kcal da dieta. O mínimo recomendado para o sódio em alimentos para cães adultos com cardiopatia é de $0,08 \%$ e $0,068 \%$ para gatos com base na matéria seca (MS) (Freeman, 2009).

Segundo Roudebush \& Keene (2010), outra recomendação de sódio para cardiopatas, devem ser restrições de $0,15 \%$ a $0,25 \%$ da MS para cães com insuficiência cardíaca leve e $0,08 \%$ a $0,15 \%$ da MS para cães com insuficiência cardíaca avançada e em gatos com insuficiência cardíaca, recomenda-se dieta com 0,07 a $0,3 \%$ de sódio.

No Brasil, de acordo com o trabalho de Nelson \& Couto (2015), o manejo alimentar de cães cardiopatas pode ser efetuado com apoio das dietas Royal Canin Early Cardiac Canine (seca) contendo $0,16 \%$ ou $40 \mathrm{mg} / 100 \mathrm{kcal}$ de sódio e Royal Canin Cardiac Canine (úmida) com 0,06\% ou 55,5 mg/100 Kcal de sódio ou dietas Hill's Prescription Diet h/d Canine (seca), com $0,08 \%$ ou $17 \mathrm{mg} / 100 \mathrm{kcal}$ de sódio, Hill's Prescription Diet $\mathrm{k} / \mathrm{d}$ Canine (seca) com $0,23 \%$ ou $52 \mathrm{mg} / 100 \mathrm{kcal}$ de sódio ou Hill's Prescription Diet k/d Canine (úmida) com $0,19 \%$ ou $40 \mathrm{mg} / 100 \mathrm{kcal}$ de sódio. Para o manejo alimentar de gatos cardiopatas, há disponíveis as dietas Royal Canin Renal Feline (seca) contendo $0,3 \%$ ou $76 \mathrm{mg} / 100 \mathrm{kcal}$ de sódio e Renal S/O Feline (úmida) com $0,12 \%$ ou 94 $\mathrm{mg} / 100 \mathrm{kcal}$ de sódio ou Hill's Prescription Diet Feline k/d (seca) contendo $0,24 \%$ ou $56 \mathrm{mg} / 100$ kcal de sódio. Para Kroll et al. (2010), uma outra alternativa seria as dietas caseiras, mas seu uso requer a participação de um profissional habilitado em nutrição.

Segundo Dove (2001), alguns nutrientes estão sendo utilizados na dieta, para prevenção e tratamento de doenças cardíacas, como os ácidos graxos essenciais, os agentes antioxidantes, a arginina, a coenzima Q10, a L-carnitina, a taurina e a vitamina $B$.

Em relação aos ácidos graxos, um tipo específico que tem recebido grande atenção por seus efeitos no sistema cardiovascular é o ômega3 ( $\omega-3)$ (Freeman, 2010; Freeman, 2000b). De acordo com Freeman (2000b) existem algumas hipóteses sobre os mecanismos de ação do $\omega-3$ no miocárdio: (1) ação direta nos canais iônicos devido à presença de sítios específicos de ligação para o $\omega-3$ na subunidade alfa dos canais de $\mathrm{Na}+$; (2) ação indireta nos canais iônicos, por meio de alterações na fluidez e na tensão superficial das membranas desses canais ou nas membranas de cardiomiócitos próximos; (3) diminuição do metabolismo celular prevenindo a ocorrência de eventos isquêmicos nos cardiomiócitos; (4) alterações na regulação do cálcio celular; (5) inibição competitiva do $\omega-3$ com enzimas produtoras de prostaglandinas; (6) possível inibição de sinalizadores da apoptose.

Para Freeman (2010), os efeitos antiinflamatórios e antiarrítmicos do $\omega-3$ podem ser benéficos na prevenção de perda de massa magra e no tratamento de arritmias diversas, como as ventriculares e a fibrilação atrial. Muitos cães com doenças cardiovasculares apresentam arritmias que podem, em algum momento, resultar em morte súbita. $\mathrm{O}$ uso de $\omega-3$ pode ser indicado mesmo antes que a insuficiência cardíaca congestiva se desenvolva nos animais cardiopatas assintomáticos (Freeman, 2000a; Freeman, 2009; Freeman, 2010). Como fonte tem sido utilizado o óleo de peixe com intuito de estabilizar a atividade elétrica do coração. Um estudo realizado na Itália observou com o tratamento uma redução 36\%$45 \%$ na morte cardíaca repentina e foi à chave para evidenciar o uso de óleo de peixe para impedir 
arritmias ventriculares fatais (Jenkins et al., 2009). A taurina é um aminoácido que desempenha a função de proteção do miocárdio e na regulação da função contrátil. Em gatos, a biossíntese de taurina a partir de seus precursores é insuficiente para suprir suas necessidades, tornando-o o então um aminoácido essencial para esta espécie e sua deficiência tem demonstrado ser a principal causa de cardiomiopatia dilatada em gatos (Chetboul \& Biourge, 2008). Os cães são capazes de sintetizar quantidades adequadas de taurina. Mas, em ambas as espécies, para pacientes cardiopatas, preconizase o uso da taurina.

Gompf (2005) relata que este aminoácido é encontrado em altas concentrações no coração. A deficiência de taurina causa o enfraquecimento do músculo do coração, o que por sua vez pode levar ao aparecimento de cardiomiopatia dilatada (CDM), a qual inclusive pode ser fatal. A CMD em gatos é uma consequência clássica da carência de taurina (Borges et al., 2016). A taurina possui efeito protetor frente a problemas do ritmo cardíaco, agindo sobre a regulação da contractilidade do coração. Além disso, os suplementos de taurina agregados ao alimento podem beneficiar cães e gatos com insuficiência miocárdica. Os animais com deficiência de taurina atestada apresentam maiores chances de melhoras quando recebem suplementação de taurina no alimento. Gatos devem receber $250-500 \mathrm{mg} / \mathrm{dia}$ de taurina e cães de 500 a $1000 \mathrm{mg} /$ dia (Hand et al., 2010). A porcentagem de taurina para alimentos com nível proteico de $28 \%$ com indicação preventiva para problemas cardíacos é de $0,1 \%$, enquanto que em alimentação indicada para tratamento de cardiopatias é de $0,19 \%$ com $25 \%$ de proteína.

As mitocôndrias são os principais locais onde ocorrem os processos aeróbicos oxidativos e a Lcarnitina é a principal responsável pelo transporte dos ácidos graxos de cadeia longa pela membrana mitocondrial, para que a oxidação e geração de energia possa ser efetivamente realizada (Kolb, 1984).

As quantidades encontradas em alimentos industrializados têm em média $200 \mathrm{mg}$ de Lcarnitina por quilo de alimento, dependendo da indicação. No manejo das cardiomiopatias preconiza-se a adição de $50 \mathrm{mg} / \mathrm{kg}$ à terapia convencional em intervalos de 8 horas, embora se trate de prática muito onerosa e, por isso, não considerada como recomendação universal (Schwantes \& Oliveira, 2006). A maneira mais prática e menos onerosa, é fornecer alimento comercial terapêutico para animais cardiopatas, os quais incluem L-carnitina em níveis mais altos em torno de $450-500 \mathrm{mg} / \mathrm{kg}$ (Carciofi, 2008).

Chetboul \& Biourge (2008) relataram em algumas raças de cães a cardiomiopatia dilatada associada à deficiência de carnitina. A suplementação com carnitina tem sido relatada ser benéfica por melhorar a produção de energia do miocárdio (Freeman, 2000a; Freeman, 2009). De acordo com os autores a maior limitação quanto ao uso é o seu alto custo.

Para Seelig \& Rosanoff (2003), o magnésio é um mineral muito importante para todas as células musculares, especialmente as cardíacas, pois participa do processo de regulação da contração e relaxamento das fibras musculares. Quando o coração se apresenta "saudável", os níveis de magnésio são maiores nesta musculatura. A deficiência deste elemento em animais pode causar arritmia e taquicardia, anormalidades no eletrocardiograma, arteriosclerose (espessamento da parede arterial), aumento da pressão arterial, e desordens de valva mitral (Kolb, 1984).

\section{Considerações Finais}

São muitos os fatores que determinam o perfeito funcionamento do músculo cardíaco, e é notória a grande importância que a nutrição tem nesse processo, onde nutrientes atuam nas mais diversas variáveis que interferem com o metabolismo do miocárdio. Por isso é necessário que a alimentação de cães e gatos cardiopatas esteja balanceada.

\section{Referências}

Assumpção, T. C. A., Hall, P. A., Pereira, E. Z., Costa, M. T., \& Júnior, D. P. 2012. Persistência do Ducto Arterioso - Revisão de Literatura. Enciclopédia Biosfera, 8(15), 1295-1325.

Azevedo, V. M. P., Albanesi Filho, F. M., Santos, M. A., Castier, M. B., \& Cunha, M. O. 2005. O papel da L-carnitina no estado nutricional e na evolução ecocardiográfica da cardiomiopatia dilatada idiopática da infância. Jornal de Pediatria, 81(5), 368-372.

Borges, O. M. M., Araújo, S. B., Azevedo, S. S., Tanikawa, A., de Santana, V. L., Silva, R. M. N., \& Souza, A. P. 2016. Estudo clínico e de fatores de risco associados às alterações cardiovasculares em cães. Pesquisa Veterinária Brasileira, 36(11), 1095-1100. 
Carciofi, A. C. 2008. Manejo nutricional do cão e do gato hospitalizado. Paper presented at the Apontamentos teóricos das disciplinas de clínica das doenças carenciais, endócrinas e metabólicas e de nutrição e alimentação de cães e gatos, Jaboticabal.

Carciofi, A. C., \& Jeremias, J. T. 2010. Progresso científico sobre nutrição de animais de companhia na primeira década do século XXI. Revista Brasileira de Zootecnia, 39(Sup. 1), 35-41.

Carr, A. P. 2008. Cardiopatia em cães e gatos idosos. In J. D. Hoskins (Ed.), Geriatria e gerontologia do cão e gato (pp. 141-166). São Paulo, São Paulo, Brasil: Roca.

Castro, M. G., Veado, J. C. C., Silva, E. F., \& Araújo, R. B. 2009. Estudo retrospectivo ecodopplercardiográfico das principais cardiopatias diagnosticadas em cães Echodopplercardiographic retrospective study of the main cardiopathies diagnosed in dogs. Arquivo Brasileiro de Medicina Veterinária e Zootecnia, 61(5), 1238-1241.

Chawla, L. S., Herzog, C. A., Costanzo, M. R., Tumlin, J., Kellum, J. A., McCullough, P. A., $\&$ Ronco, C. 2014. Proposal for a functional classification system of heart failure in patients with end-stage renal disease: proceedings of the acute dialysis quality initiative (ADQI) XI workgroup. Journal of the American College of Cardiology, 63(13), 1246-1252.

Chetboul, V., \& Biourge, V. 2008. Acquired cardiovascular diseases in cats: the influence of nutrition. In P. Pibot, V. Biourge \& D. Elliott (Eds.), Encyclopedia of feline clinical nutrition (pp. 323-355). Aimargues: Aniwa SAS.

Darke, P. G. G., Bonagura, J. D., \& Kelly, D. F. 2000. Atlas ilustrado de cardiologia veterinária. São Paulo, São Paulo, Brasil: Manole.

Dove, R. S. 2001. Nutritional therapy in the treatment of heart disease in dogs. Alternative Medicine Review, 6(Sup. 1), 38-45.

Evangelista, J. 2005. Tecnologia de alimentos. São Paulo, São Paulo, Brasil: Atheneu.

Freeman, L. M. 2000a. Nutritional modulation of cardiac disease. Waltham Focus, 10(2).

Freeman, L. M. 2009. Nutritional management of heart disease. In J. D. Bonagura \& D. C. Twedt (Eds.), Kirk's current veterinary therapy (pp. 704-708). St. Louis, USA: Saunders.
Freeman, L. M. 2010. Beneficial effects of omega3 fatty acids in cardiovascular disease. Journal of Small Animal Practice, 51(9), 462-470.

Freeman, M. P. 2000b. Omega-3 fatty acids in psychiatry: a review. Annals of Clinical Psychiatry, 12(3), 159-165.

Gompf, R. E. 2005. Nutritional and herbal therapies in the treatment of heart disease in cats and dogs. Journal of the American Animal Hospital Association, 41(6), 355-367.

Hand, M. S., Thatcher, C. D., Remillard, R. L., Roudebush, P., \& Novtony, B. J. 2010. Small animal clinical nutrition: Mark Morris Institute Topeka.

Husain-Syed, F., McCullough, P. A., Birk, H.-W., Renker, M., Brocca, A., Seeger, W., \& Ronco, C. 2015. Cardio-pulmonary-renal interactions: a multidisciplinary approach. Journal of the American College of Cardiology, 65(22), 2433-2448.

Jenkins, D. J. A., Sievenpiper, J. L., Pauly, D., Sumaila, U. R., Kendall, C. W. C., \& Mowat, F. M. 2009. Are dietary recommendations for the use of fish oils sustainable? Canadian Medical Association Journal, 180(6), 633-637.

Kolb, E. 1984. Fisiologia veterinária. Rio de Janeiro: Koogan.

Kroll, F. S. A., Champion, T., Lopes, F., Brunetto, M. A., \& Carciofi, A. C. 2010. A importância do sódio no manejo nutricional de cães e gatos cardiopatas. Medvep - Revista Científica de Medicina Veterinária - Pequenos Animais e Animais de Estimação, 8(27), 608-614.

Little, C. J., Julu, P. O., Hansen, S., \& Reid, S. W. 2005. Non-invasive real-time measurements of cardiac vagal tone in dogs with cardiac disease. The Veterinary Record, 156(4), 101-105.

Morais, H. A. 2005. Pathophysiology of heart failure and clinical evaluation of cardiac function. In S. J. Ettinger \& E. C. Feldman (Eds.), Textbook of Veterinary Internal Medicine (pp. 692-712). St. Louis, Missouri, USA: Saunders.

Nelson, R. W., \& Couto, C. G. 2015. Medicina interna de pequenos animais. Amsterdan: Elsevier Editora.

Randall, D., Burggren, W., \& French, K. 2000. Fisiologia Animal Mecanismos e Adaptações. Rio de Janeiro, Brasil: Guanabara Koogan.

Roudebush, P., \& Keene, B. W. 2010. Cardiovascular disease. In M. S. Hand, C. D. Thatcher, R. L. Remillard, P. Roudebush \& B. 
J. Novotny (Eds.), Small animal clinical nutrition (pp. 733-763). Kansas, USA: Mark Morris Institute.

Saad, F. M. O. B., \& França, J. 2010. Alimentação natural para cães e gatos. Revista Brasileira de Zootecnia, 39(1), 52-59.

Santos Júnior, E. R., Melo, A. N., \& Wischral, A. 2007. Fisiopatologia da insuficiência cardíaca e o uso do maleato de enalapril em cães. Ciência Veterinária nos Trópicos, 10(1), 1-8.

Schwantes, V. C., \& Oliveira, S. T. 2006. Cardiomiopatia do Boxer: revisão de literatura. Clínica Veterinária, 11(64), 48-58.

Seelig, M. S., \& Rosanoff, A. 2003. The magnesium factor. USA: Penguin.

Silveira, J. A. M., Morais, G. B., Macambira, K. D. S., Francisco Júnior, A. F. X., Pessoa, N. O., Costa, P. P. C., \& Evangelista, J. S. A. M. 2015. Cardiomiopatia hipertrófica felina: aspectos relevantes. Revista Brasileira de Higiene e Sanidade Animal, 9(3), 465-476.

Slupe, J. L., Freeman, L. M., \& Rush, J. E. 2008. Association of body weight and body condition with survival in dogs with heart failure.
Journal of Veterinary Internal Medicine, 22(3), 561-565.

Teerlink, J. R. 1996. Neurohumoral mechanisms in heart failure: a central role for the reninangiotensin system. Journal of Cardiovascular Pharmacology, 27, 1-8.

Tilley, L. P., \& Goodwin, J.-K. 2002. Manual de cardiologia para cães e gatos. In K. N. Strickland (Ed.), Livro Manual de Cardiologia para Cães e Gatos (pp. 323-345): Roca, São Paulo, Brasil.

Zaine, L., Monti, M., Vasconcellos, R. S., \& Carciofi, A. C. 2014. Nutracêuticos imunomoduladores com potencial uso clínico para cães e gatos. Semina: Ciências Agrárias, 35(4), 2513-2530.

Recebido: 16 Setembro 2018

Aprovado: 30 Outubro 2018.

Publicado: 27 Novembro 2018.

Licenciamento: Este artigo é publicado na modalidade Acesso Aberto sob a licença Creative Commons Atribuição 4.0 (CC-BY 4.0), a qual permite uso irrestrito, distribuição, reprodução em qualquer meio, desde que o autor e a fonte sejam devidamente creditados. 